\title{
A Novel Adaptive OFO-OFDM Modulation for Visible Light Communication
}

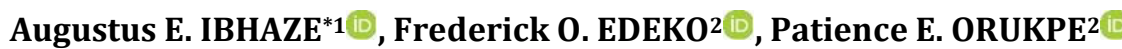 \\ ${ }^{1}$ Department of Electrical and Electronics Engineering, University of Lagos, Lagos, 100213, Nigeria \\ ${ }^{2}$ Department of Electrical/Electronic Engineering, University of Benin, Benin City, 300283, Nigeria
}

(Alınış / Received: 25.10.2020, Kabul / Accepted: 24.05.2021, Online Yayınlanma / Published Online: 15.08.2021)

\section{Keywords}

Demodulation,

Intensity Modulation,

Modulation,

OFDM,

Optical Modulation,

Visible Light Communication

\begin{abstract}
Radio frequency techniques have been deployed in the past time for wireless communication and following its spectral crunch and limited throughput, the visible light spectrum with enormously large bandwidth and potentials for high throughput is being investigated in this work. This study is aimed at investigating and modeling a signal conditioning scheme in the visible light spectrum in view of enhancing the throughput of the network. To effectively enhance communication throughput, a possible solution is to deploy multicarrier techniques. In this work, an adaptive Optimized Flipped Optical (OFO) OFDM is proposed for improved throughput using Lagrange Multiplier and Broyden Fletcher Goldfarb Shanno Algorithm (BFGSA). The Lagrange Multiplier technique was used to formulate the model for the optimization of the throughput constrained by the bit error rate (BER) and the total subcarrier transmit power whereas the BFGSA was used for the estimation of the approximation to the Hessian matrix for the computation of the optimal throughput value. Results showed improved spectral efficiency in favor of the proposed algorithm when compared with the conventional schemes. Further validations revealed the performance superiority of the proposed algorithm when compared with Castel, Wyglinski and Bedeer algorithms under comparable operating conditions for a given average signal to noise ratio (SNR).
\end{abstract}

\section{Görünür Işık İletişimi için Yeni Bir Adaptif OFO-OFDM Modülasyonu}

\author{
Keywords \\ Demodülasyon, \\ Yoğunluk Modülasyonu, \\ Modülasyon, \\ OFDM, \\ Optik Modülasyon, \\ Görünür Işılk İletişimi
}

\begin{abstract}
Özet: Radyo frekansı teknikleri Geçmiște kablosuz iletișim için konușlandırılmıș ve spektral sıkıntısını ve sınırlı iş hacmini takiben, son derece büyük bant genişliğine sahip görünür ışık spektrumu ve yüksek verimlilik potansiyeli bu çalışmada araştırılmaktadır. $\mathrm{Bu}$ çalışma, ağın verimini artırmak için görünür $1 s ̧ \mathrm{k}$ spektrumunda bir sinyal koşullandırma şemasını araştırmayı ve modellemeyi amaçlamaktadır. İletişim verimini etkili bir şekilde artırmak için olası bir çözüm, çoklu taşıyıcı tekniklerini kullanmaktır. Bu çalışmada, Lagrange Multiplier ve Broyden Fletcher Goldfarb Shanno Algorithm (BFGSA) kullanılarak geliştirilmiş verim için uyarlanabilir bir Optimize Edilmiş Ters Optik (OFO) OFDM önerilmiştir. Lagrange Çarpanı tekniği, bit hata oranı (BER) ve toplam alt taşıyıcı iletim gücü ile sınırlandırılan verimin optimizasyonu için modeli formüle etmek için kullanılırken, BFGSA, aşağıdaki hesaplamalar için Hessian matrisine yaklaşımın tahmini için kullanılmıştır. Sonuçlar, geleneksel şemalarla karşılaştırıldığında önerilen algoritmanın lehine gelişmiş spektral verimlilik gösterdi. Diğer doğrulamalar, belirli bir ortalama sinyal-gürültü oranı (SNR) için karşılaştırılabilir çalışma koşulları altında Castel, Wyglinski ve Bedeer algoritmalarıyla karşılaştırıldığında önerilen algoritmanın performans üstünlügünü ortaya koydu.
\end{abstract}

\section{Introduction}

Wireless communication has become the mainstay for global integration across diverse metrics of technology, economies, governance and institutions to mention a few. With the advent of technology revolution alongside global population density, the existing radio frequency spectrum is encumbered by spectral congestion and overutilization and as such demands the integration of alternative spectrum for 
seamless wireless communication coverage and capacity. As demand for wireless communication services increase, the need to deploy high speed wireless communication infrastructure becomes necessary. With a quick overview of the existing communication technologies, first generation technology could only achieve $2.4 \mathrm{kbps}$ data rate [1] in comparison with the fourth generation technology with a data rate of $30 \mathrm{Mbps}$ [2]. In view of the rising demand of the present data hungry society, fifth generation technology has been proposed to achieve at least $1 \mathrm{Gbps}$ [3]. In order to make the ecosystem a more amiable place with effective and efficient communication infrastructure for seamless integration, this research undertaking is therefore dosed with the motive of investigating a possible solution for high data rate integration within the wireless communication framework.

Visible light frequency spectrum can serve as a complementary band to the over utilized, congested and consequently exhausted radio frequency spectrum to serve as a means to capacity upgrade of the existing wireless communication network. The spectrum is characterized by large frequency bandwidth spanning the range of $430 \mathrm{THz}$ to $790 \mathrm{THz}$ of the electromagnetic wave spectrum and as such has the potentials for high throughput transmission [4]. Despite these merits, the nature of the frequency band requires some level of ingenuity in signal processing as techniques suitable for radio frequency design do not apply directly. This is so because visible light signals have to be intensity modulated on transmission and directly detected on reception thereby placing a limit on signal processing. This limit is partly surmounted by achieving a nonnegative real valued unipolar signal contrary to the complex valued bipolar signal processed for radio frequency spectrum applications [4].

In order to achieve high throughput/data rates, single carrier modulation schemes in time past were not able to mitigate the frequency selective related problem of the transmission process [5] which arises from the highly dispersive and multipath driven wireless channel. This made the Orthogonal Frequency Division Multiplexing (OFDM) technique ideal for mitigating frequency selectivity $[6,7]$ while improving transmission data rate through diverse multicarrier and spatial diversity techniques $[9,10]$ whereas single carrier systems deployed the use of equalizers [8]. In recent past, single carrier with frequency domain equalization (SC-FDE) has been investigated to have similar properties with OFDM as it eliminates the high peak-to-average power ratio (PAPR) associated with OFDM [11, 12]. Although, these modulation schemes have been well developed for radio frequency applications, the limited bandwidth of the radio frequency spectrum has placed a limit on the data rate achievable within its domain and as such alternative frequency bands such as the visible light frequency spectrum is now being investigated. Coupled with the inherent larger bandwidth property of the visible light spectrum is the capability for higher data rate by adapting the well-developed radio frequency techniques for visible light communication (VLC) deployment. A fundamental challenge in processing signals at visible light spectrum is the fact that the signal has to be real valued and unipolar as opposed to radio frequency signals that are complex and bipolar. This property of the signal therefore requires some level of ingenuity in processing and channel estimations since existing ideologies do not apply directly. To combine the functionality of alternate frequency band such as visible light spectrum and the welldeveloped/congested radio frequency band for improved data rates, signal transmission at visible light spectrum, a critical factor in OFDM applications has to be considered $[13,14]$ while developing high performance channel models for high throughput.

Multicarrier scheme has been investigated by $[15,16$, $17,18,19]$ for improved user experience in terms of spectral efficiency, power efficiency and higher throughput. In comparison with its single carrier counterpart, OFDM is able to eliminate inter symbol interference (ISI) and inter carrier interference (ICI) via the implementation of guard time between adjacent symbols and ensuring orthogonality of the subcarriers respectively. Since spectral wastage alongside channel impairments arising from the dispersive effect of the communication pathway, form major challenge in single carrier technique adoption, multicarrier technique with orthogonal frequency division multiplexing (OFDM) is proposed to resolve some of these challenges while imposing new ones. It is to be noted that the traditional single carrier techniques [20] optimized either with frequency domain linear equalization [21] or decision feedback equalization [22] could provide comparable performance estimate with multicarrier techniques such as OFDM at low SNR whereas at high SNR, there is a tradeoff between performance, design complexity, signal processing and propagation scenario. In terms of achievable higher data rates, despite performance comparability under channel loading conditions, OFDM technique has shown optimum performance relative to increasing SNR [23] having bottlenecks in high PAPR and consequent nonlinearity effect of the transmitter front-end. These bottlenecks lead to high bit error rates and performance degradation in radio frequency applications. While this supposition has been an academic and industrial problem under research investigation for quite some time, the adoption of multicarrier techniques with OFDM in VLC leads to the direct adoption of these problems. The major consideration now follows directly from measures required for the adaptation of multicarrier schemes in VLC owing to the peculiarity of the processed signal, (that is, requiring intensity modulation and 
direct detection) for high throughput under increasing SNR and low bit error rates.

OFDM application is such that the transmission ideology is based on the division of the given bandwidth into $\mathrm{N}$ subcarriers modulated in the frequency domain by inverse Fast Fourier Transform (IFFT). If the given $\mathrm{N}$ subcarriers are assigned in a single OFDM block, the transmitted serial bit sequence is first converted to parallel bit sequence and then mapped to a fraction of the $\mathrm{N}$ subcarriers depending on the modulation format. The modulated OFDM block is then reconstructed in such a way that part of the modulated subcarriers are used to convey the information symbol, whereas some are set to zero to avoid complex harmonics and dc components being degrading signal components while others are used to impose Hermitian symmetry which equips the transmitter with the capability to generate time domain real valued signals for VLC applications. After IFFT operation on the modulated signal, guard time (cyclic prefix) is introduced between symbols to help eliminate ISI while maintaining subcarrier orthogonality. This is done before reconverting the parallel bit sequence to serial combination. The eventual bit sequence is then fed into a digital-toanalog converter which results in a bipolar electrical signal lacking the fundamental property of initiating intensity modulation required to drive the LED transmitter [24].

To adapt these salient features of OFDM modulation in visible light applications, the bipolar signal has to be converted to unipolar signal to enable intensity modulation [4]. This particular supposition has become a research problem as means to achieve the OFDM unipolar signal without sacrificing the ultimate performance is currently being investigated. According to [25], a direct current (DC) bias was added to the bipolar OFDM signal to generate a unipolar OFDM signal. In the proposition, the negative peaks of the biased signals were clipped to zero to achieve the unipolar signal for intensity modulation. The resulting direct current optical (DCO) OFDM was quite spectrally efficient, built with minimal design complexity but was lacking in power efficiency. This loss in power efficiency was attributed to the fact that higher DC bias was required to clip the negative amplitudes to zero thereby placing a limit on the achievable DCO-OFDM performance due to clipping distortion.

Attempts to improve on the optical OFDM performance led to modifications of the DCO-OFDM in [26] where the authors proposed a power efficient OFDM scheme. In this case, the OFDM signal was clipped at zero without need for direct current bias. Hence, data was conveyed using odd subcarriers alone while sacrificing the even subcarriers to cater for clipping distortion. This idea was borne from the fact that the two-half cycles of the samples have equivalent amplitude with opposite polarity so that suppressing one-half will have no negative impact on the transmitted data. This asymmetrically clipped optical (ACO) OFDM achieved improved power efficiency at the expense of spectral wastage since half of the subcarriers were clipped to cater for clipping distortion. Hence, ACO OFDM could achieve half DCO OFDM spectral efficiency.

In the work of [27], unipolar (U) OFDM was proposed. The authors combined the two-halves of the OFDM samples to generate the unipolar signal. Rather than suppress a part of the samples as in the case of ACO OFDM, the sample with opposite polarity was flipped to achieve unipolar signal. This operation resulted in the extension of the OFDM block length making it twice as large. This enabled the transmission of the negative peaks thereby eliminating the need for clipping. The U-OFDM or Flip OFDM was able to achieve improved power efficiency at the expense of spectral efficiency. The achievable spectral efficiency is half of DCO OFDM efficiency since the OFDM block length is twice as large in the case of Flip-OFDM. The tradeoff between spectral efficiency and power efficiency leads to either performance enhancement or degradation in relation to SNR and BER performance which has led to the need for further investigation being carried out in this work. This research work will proffer solution to the identified shortcomings discussed in these related works of VLC systems by developing an optimized FLIP scheme for improved spectral and power efficiency. The optimization of the preferred conventional optical scheme (FLIP) is so achieved by defining its objective function which is the transmission rate subject to the transmission power and the channel effect on the transmitted bits (bit error rate). The adaptation of the transmission rate relative to the transmission power and BER results in a dynamic scheme that guarantees improved throughput at increasing average signal-to-noise ratio. The remaining part of this article is organized as follows: Section 2 presents the mathematical models depicting the relationship between BER and SNR of the conventional signal conditioning schemes whereas Section 3 provides the modeling of the proposed adaptive signal conditioning scheme. Section 4 presents the results and validation of the findings with the conclusion drawn in Section 5.

\section{Method}

Following the deductions made in the literature relative to the conventional DCO-OFDM, ACO-OFDM and FLIP-OFDM, it is therefore logical to seek measures for improving upon the BER and spectral performances of the FLIP scheme since it surpasses the DCO and ACO schemes [28, 29]. In view of the important parameters of the optical OFDM scheme; transmit power, throughput, bit error rate and signal to noise ratio, it is imperative to optimize the scheme such that the throughput is maximized at low 
transmit power. This therefore leads to the formulation of an optimization problem of maximizing the throughput while adaptively minimizing the transmit power subject to the degrading effects of the noisy channel resulting in BER. Since higher transmit power could lead to power inefficiency, transmitting at lower power will further improve upon the overall system performance. Hence, defining the power threshold for individual subcarrier will again constrain the objective function of the optimization problem in achieving a suitable solution. In contrast to the conventional modulation schemes (DCO-, ACO-, and FLIP-), adaptive modulation schemes provides parameters that can be modified relative to the fading channel; some of which are the data rate (throughput), transmit power, constellation size and symbol rate among others [30]. The fundamental query that therefore arises is to which of the parameters should be adapted for improved performance. Considering the trend in data usage and the growing data rate requirement, the need to optimize the effective throughput (data rate) has become paramount. The transmission parameters that can be optimized include the transmission data rate (throughput), power and bit error rate. The transmission parameters so identified includes the individual subcarrier throughput $b_{\gamma, F L I P}$ as defined in the objective function, the individual subcarrier transmit power $P_{\gamma, F L I P}$ as defined in the power constraint and the bit error rate (BER) which is a function of the channel impact on the transmission process. For the sake of optimization/adaptation, the initial (input) parameters were defined in Equation (1) while the aftermath of the optimization process engendered the output throughput as $b_{\gamma, \text { final }}$.

To derive the optimal transmission rate, power and BER adaptation that will maximize the throughput of the FLIP scheme subject to the average BER and power constraint, the objective function to be optimized is defined as:

Maximize:

$$
b_{t h r u}=\sum_{\gamma=1}^{N / 2-1} b_{\gamma, F L I P}
$$

subject to:

and

$$
B E R_{\text {ave }}=\frac{\sum_{\gamma=1}^{N / 2-1} b_{\gamma, F L I P} B E R_{\gamma, F L I P}}{\sum_{\gamma=1}^{N / 2-1} b_{\gamma, F L I P}} \leq B E R_{\text {thres }}
$$

$$
P_{T}=\sum_{\gamma=1}^{N / 2-1} P_{\gamma, F L I P} \leq P_{\text {thres }}
$$

where $b_{\text {thru }}$ is the throughput, $b_{\gamma, F L I P}$ is the individual subcarrier throughput, $\gamma$ is the subcarrier index $(1 \leq$ $\gamma \leq N / 2-1), N / 2-1$ is the total number of subcarriers available for information bit transmission, $B E R_{\text {ave }}$ is average BER, $B E R_{\gamma, F L I P}$ is the $\mathrm{BER}$ contribution of individual subcarrier, $B E R_{\text {thres }}$ is the defined threshold BER value, $P_{T}$ is the total transmit power, $P_{\gamma, F L I P}$ is the power required to transmit information bits over a given $\gamma$ subcarrier and $P_{\text {thres }}$ is the defined threshold total transmit power.

For high throughput applications, the fixed order of MQAM signal conditioning techniques have been severally deployed for OFDM implementation as attested to in the literature [24, 31, 32, 33]. As such, for ease of validation, MQAM signal conditioning techniques will be investigated for adaptive signal conditioning in the visible light frequency spectrum in this research undertaking. An approximation to the BER of the MQAM with respect to the power distribution of the individual subcarrier index $\gamma$ is given by $[34,35]$ :

$$
B E R_{\gamma, F L I P} \approx 0.2 \exp \left(-1.6 \frac{P_{\gamma, F L I P}}{2^{b_{\gamma, F L I P}-1}} \xi_{\gamma}\right)
$$

where $\xi_{\gamma}$ is the channel-to-noise ratio defined in terms of the ratio of the square of the channel gain per subcarrier $H_{\gamma}$ to the variance $\sigma_{\gamma}^{2}$ of the additive white Gaussian noise (AWGN) for a sufficiently large number of subcarriers $N$, given by:

$$
\xi_{\gamma}=\frac{\left|H_{\gamma}\right|^{2}}{\sigma_{\gamma}^{2}}
$$

where $\left|H_{\gamma}\right|^{2}$ is a measure of signal attenuation with respect to transmitter - receiver separation distance accounting for the pathloss.

By combining (1) and (2), we obtain:

Maximize:

subject to:

$$
b_{\text {thru }}=\sum_{\gamma=1}^{N / 2-1} b_{\gamma, \text { FLIP }}
$$

$$
\begin{array}{r}
B E R_{\text {ave }}=\sum_{\gamma=1}^{N / 2-1} b_{\gamma, F L I P} B E R_{\gamma, F L I P} \leq \\
B E R_{\text {thres }} \sum_{\gamma=1}^{N / 2-1} b_{\gamma, F L I P}
\end{array}
$$

and

$$
P_{T}=\sum_{\gamma=1}^{N / 2-1} P_{\gamma, F L I P} \leq P_{\text {thres }}
$$

such that

Maximize:

$$
b_{\text {thru }}=\sum_{\gamma=1}^{N / 2-1} b_{\gamma, F L I P}
$$

subject to:

$$
\begin{aligned}
& B E R_{\text {ave }}= \\
& 0.2 \sum_{\gamma=1}^{N / 2-1} b_{\gamma, \text { FLIP }} \exp \left(-1.6 \frac{P_{\gamma, F L I P}}{2^{b_{\gamma, F L I P}-1}} \xi_{\gamma}\right)- \\
& B E R_{\text {thres }} \sum_{\gamma=1}^{N / 2-1} b_{\gamma, F L I P} \leq 0
\end{aligned}
$$

and

$$
P_{T}=\sum_{\gamma=1}^{N / 2-1} P_{\gamma, F L I P}-P_{\text {thres }} \leq 0
$$

For ease of analysis, (5) can be rewritten as:

$$
\begin{gathered}
\underset{b_{\gamma, F L I P}}{\operatorname{Maximize}} F(\boldsymbol{b})=\tau \sum_{\gamma=1}^{N / 2-1} b_{\gamma, F L I P} \\
\text { subject to } c_{i}(\boldsymbol{b}, \boldsymbol{p}) \leq 0 \quad \forall \quad 1 \leq i \leq 2
\end{gathered}
$$


where $F(\boldsymbol{b})$ is the objective function defining the throughput such that $\boldsymbol{b}=\left[b_{1}, b_{2}, \ldots, b_{N / 2-1}\right]^{T}$ describes the bit vector distribution, $\tau$ is a constant whose value defines the relevance of the objective function normalized to $\tau=1, c_{i}(\boldsymbol{b}, \boldsymbol{p})$ defines the linear combination of the constraints and $i$ defines the total number of constraints imposed on the objective function such that:

$$
\begin{aligned}
& c_{i}(\boldsymbol{b}, \boldsymbol{p})= \\
& \left\{\begin{array}{cc}
0.2 \sum_{\gamma=1}^{N / 2-1} b_{\gamma, F L I P} \exp \left(-1.6 \frac{P_{\gamma, F L I P}}{2^{b_{\gamma, F L I P}-1}} \xi_{\gamma}\right)- \\
B E R_{\text {thres }} \sum_{\gamma=1}^{N / 2-1} b_{\gamma, F L I P} \leq 0 & \forall i=1 \\
\sum_{\gamma=1}^{N / 2-1} P_{\gamma, F L I P}-P_{\text {thres }} \leq 0 & \forall i=2
\end{array}\right.
\end{aligned}
$$

Hence, the problem of (6) can be solved by Lagrange multiplier such that the relationship between the gradient of the objective function and the gradient of the constraints is drawn by the introduction of the Lagrange multiplier $\lambda$ such that:

$$
\nabla F(\boldsymbol{b})=\lambda \nabla c_{i}(\boldsymbol{b}, \boldsymbol{p})
$$

where $\lambda=\left[\lambda_{i}\right]^{T} \forall 1 \leq i \leq 2$ is the Lagrange multiplier vector

The implication of the Lagrange multiplier is such that it defines the stationary point at which the objective function and the constraints are tangent to each other such that their respective gradient vectors are parallel to each other. Since setting the gradient of a given function to zero enables the realization of its maximum or minimum value and being that the gradient of the objective function may not necessarily be equal to the gradient of the constraints, the Lagrange multiplier is therefore imposed to enforce equality.

Considering (6), the inequality constraints are converted to equality constraints by introducing nonnegative slack variable, $S_{i}^{2} \quad \forall 1 \leq i \leq 2$ [36], so that:

$C_{i}(\boldsymbol{b}, \boldsymbol{p}, \boldsymbol{s})=c_{i}(\boldsymbol{b}, \boldsymbol{p})+S_{i}^{2}=0 \quad \forall 1 \leq i \leq 2$

where $s=\left[S_{i}^{2}\right]^{T} \quad \forall 1 \leq i \leq 2$ is the slack variable vector.

Equation (8) can be rewritten as:

$$
\nabla F(\boldsymbol{b})=\lambda \nabla C_{i}(\boldsymbol{b}, \boldsymbol{p}, \boldsymbol{s})
$$

The Lagrangian function $\mathcal{L}$ follows directly from (10) as:

$$
\mathcal{L}(\boldsymbol{b}, \boldsymbol{p}, \boldsymbol{s}, \lambda)=F(\boldsymbol{b})-\lambda C_{i}(\boldsymbol{b}, \boldsymbol{p}, \boldsymbol{s})
$$

By combining (6), (7) and (11), the Lagrangian function required to maximize the throughput is derived:

$$
\begin{gathered}
\mathcal{L}(\boldsymbol{b}, \boldsymbol{p}, \boldsymbol{s}, \boldsymbol{\lambda})= \\
\tau \sum_{\gamma=1}^{N / 2-1} b_{\gamma, F L I P}- \\
\lambda_{1}\left[0.2 \sum_{\gamma=1}^{N / 2-1} b_{\gamma, F L I P} \exp \left(-1.6 \frac{P_{\gamma, F L I P}}{2^{b_{\gamma, F L I P}-1}} \xi_{\gamma}\right)-\right. \\
\left.B E R_{\text {thres }} \sum_{\gamma=1}^{N / 2-1} b_{\gamma, F L I P}+S_{1}^{2}\right]- \\
\lambda_{2}\left[\sum_{\gamma=1}^{N / 2-1} P_{\gamma, F L I P}-P_{\text {thres }}+S_{2}^{2}\right]
\end{gathered}
$$

The stationary point can be obtained by equating the gradient of the Lagrangian function to zero. That is, $\nabla \mathcal{L}(\boldsymbol{b}, \boldsymbol{p}, \boldsymbol{s}, \lambda)=0$, which is given by:

$$
\nabla \mathcal{L}(\boldsymbol{b}, \boldsymbol{p}, \boldsymbol{s}, \lambda)=0
$$

Such that:

$$
\begin{aligned}
& \nabla \mathcal{L}(\boldsymbol{b})=\tau-\lambda_{1}\left[0.2 \exp \left(\frac{-1.6 P_{\gamma, F L I P} \xi_{\gamma}}{2^{b_{\gamma, F L I P}-1}}\right)+\right. \\
& 0.32 \log _{e} 2 \frac{P_{\gamma, F L I P} \xi_{\gamma} b_{\gamma, F L I P} 2^{b_{\gamma, F L I P}}}{\left(2^{b_{\gamma, F L I P}-1}\right)^{2}} \exp \left(\frac{-1.6 P_{\gamma, F L I P} \xi_{\gamma}}{2^{b_{\gamma, F L I P}-1}}\right)- \\
& \left.B E R_{\text {thres }}\right]=0 \\
& \nabla \mathcal{L}(\boldsymbol{p})=\lambda_{1}\left[\frac{0.32 \xi_{\gamma} \sum_{\gamma=1}^{N / 2-1} b_{\gamma, F L I P}}{2^{b_{\gamma, F L I P}-1}} \exp \left(\frac{-1.6 P_{\gamma, F L I P} \xi_{\gamma}}{2^{b_{\gamma, F L I P}-1}}\right)\right]- \\
& \lambda_{2}=0
\end{aligned}
$$$$
\nabla \mathcal{L}\left(\lambda_{1}\right)=
$$$$
-0.2 \sum_{\gamma=1}^{N / 2-1} b_{\gamma, F L I P} \exp \left(-1.6 \frac{P_{\gamma, F L I P}}{2^{b_{\gamma, F L I P}-1}} \xi_{\gamma}\right)+
$$$$
B E R_{\text {thres }} \sum_{\gamma=1}^{N / 2-1} b_{\gamma, F L I P}-S_{1}^{2}=0
$$$$
\nabla \mathcal{L}\left(\boldsymbol{\lambda}_{\mathbf{2}}\right)=-\sum_{\gamma=1}^{N / 2-1} P_{\gamma, F L I P}+P_{\text {thres }}-S_{2}^{2}=0
$$$$
\nabla \mathcal{L}\left(\boldsymbol{s}_{1}\right)=-2 S_{1} \lambda_{1}=0
$$$$
\nabla \mathcal{L}\left(\boldsymbol{s}_{2}\right)=-2 S_{2} \lambda_{2}=0
$$

Based on the fact that the maximization of a function $F(\boldsymbol{b})$ is equivalent to the minimization of the function $-F(\boldsymbol{b})$ [37], the optimization problem in (6) can then be reformulated as:

$$
\begin{array}{r}
\underset{b_{\gamma, F L I P}}{\text { Minimize }} F(\boldsymbol{b})=-\tau \sum_{\gamma=1}^{N / 2-1} b_{\gamma, F L I P} \\
\text { subject to } c_{i}(\boldsymbol{b}, \boldsymbol{p}) \leq 0 \quad \forall \quad 1 \leq i \leq 2
\end{array}
$$

such that the gradient of the Lagrangian function becomes:

$$
\begin{aligned}
\nabla \mathcal{L}(\boldsymbol{b})= & -\tau+\lambda_{1}\left[0.2 \exp \left(\frac{-1.6 P_{\gamma, F L I P} \xi_{\gamma}}{2^{b_{\gamma, F L I P}-1}}\right)+\right. \\
& 0.32 \log _{e} 2 \frac{P_{\gamma, F L I P} \xi_{\gamma} b_{\gamma, F L I P} 2^{b_{\gamma, F L I P}}}{\left(2^{b_{\gamma, F L I P}-1}\right)^{2}} \exp \left(\frac{-1.6 P_{\gamma, F L I P} \xi_{\gamma}}{2^{b_{\gamma, F L I P}-1}}\right)- \\
& \left.B E R_{\text {thres }}\right]=0 \\
\nabla \mathcal{L}(\boldsymbol{p})= & -\lambda_{1}\left[\frac{0.32 \xi_{\gamma} \sum_{\gamma=1}^{N / 2-1} b_{\gamma, F L I P}}{2^{b_{\gamma, F L I P}-1}} \exp \left(\frac{-1.6 P_{\gamma, F L I P} \xi_{\gamma}}{2^{b_{\gamma, F L I P}-1}}\right)\right]+ \\
\lambda_{2}= & 0
\end{aligned}
$$




$$
\begin{gathered}
\nabla \mathcal{L}\left(\boldsymbol{\lambda}_{1}\right)=0.2 \sum_{\gamma=1}^{N / 2-1} b_{\gamma, F L I P} \exp \left(-1.6 \frac{P_{\gamma, F L I P}}{2^{b_{\gamma, F L I P}-1}} \xi_{\gamma}\right)- \\
B E R_{\text {thres }} \sum_{\gamma=1}^{N / 2-1} b_{\gamma, F L I P}+S_{1}^{2}=0 \\
\nabla \mathcal{L}\left(\boldsymbol{\lambda}_{2}\right)=\sum_{\gamma=1}^{N / 2-1} P_{\gamma, F L I P}-P_{\text {thres }}+S_{2}^{2}=0 \\
\nabla \mathcal{L}\left(\boldsymbol{s}_{\mathbf{1}}\right)=2 S_{1} \lambda_{1}=0 \\
\nabla \mathcal{L}\left(\boldsymbol{s}_{2}\right)=2 S_{2} \lambda_{2}=0
\end{gathered}
$$

The gradient of the function in (16) provides a system of equations $\mathrm{f}\left(\omega_{j}\right)$ with $\omega_{j}$ unknowns where $\omega_{j}=[\boldsymbol{b}, \boldsymbol{p}, \boldsymbol{s}, \lambda]$ and $j$ order of equations. Such that, $S_{1}=\lambda_{1}=0$ and $S_{2}=\lambda_{2}=0$. By way of extension, application of these derived parameters $\left\{S_{i}=\lambda_{i}=0 \quad \forall 1 \leq i \leq 2\right\}$ in the system of equations derived in (16) will result in an undetermined system having no unique solution. Hence, the system of equations in (16) cannot be solved analytically. Therefore, unconstrained optimization techniques (numerical approach) will be attempted to arrive at a unique solution.

Since the first order derivative test being the gradient of the Lagrangian function determines the stationary point (slope of the tangent), the maximum change of the value of the given function usually lies along the direction of the gradient. The gradient therefore provides sufficient details to locate the maximum value of the Lagrangian function. Since the search direction along the gradient of a function in a constrained optimization problem could lead to an infeasible region, to identify the point that coincides exactly with the optimal value, a second order derivative test could be conducted which in essence results in the Hessian Matrix such that the derivative test of an unconstrained formulation applies [38, 39]. In relation to the formulation modified in (15), the Hessian Matrix is given as;

$$
[H]=\nabla^{2} \mathcal{L}(\boldsymbol{b}, \boldsymbol{p}, \boldsymbol{s}, \lambda)
$$

Since the derivative test converts the constrained optimization problem into a form in which unconstrained optimization techniques could be applied, the Hessian Matrix could be estimated by using any of the known unconstrained techniques for quick convergence. The various unconstrained techniques in the literature are the steepest descent, Newton, Levenberg-Marquardt, Davidon-FletcherPowell (DFP) and Broyden-Fletcher-Goldfarb-Shanno (BFGS), [35, 38, 40]. Although the steepest decent approach converges when $\omega$ is quite far from its optimal solution $\omega_{\text {opt }}$, the Newton technique performs optimally when $\omega$ is closest to $\omega_{\text {opt }}$. Levenberg-Marquardt is a hybrid technique combining the features of steepest decent and Newton approach but shows some level of sluggishness in converging to its optimum value $\omega_{\text {opt }}$. Whereas Davidon Fletcher Powell technique converges to the inverse Hessian matrix, the Broyden-Fletcher Goldfarb Shanno converges to the Hessian matrix with less error [40]. It therefore suffices to adopt BFGS to obtain the maximization of the throughput since it will converge at the Hessian matrix already derived in (17) with improved accuracy. This algorithm has been carefully chosen as no known literature at the time of this study had adopted same for similar studies.

The BFGS method utilizes an optimal step length component $\alpha_{j}$ to control the search direction in each iteration $j$, depending on the nearness to the optimal value $\omega_{\text {opt }}$. Iteration starts with an initial point $\omega_{o}$ and initial step size $h_{o}$ for which a series of $\omega_{j}$ points $\omega_{1}, \omega_{2}, \omega_{3}, \ldots$ that converges towards a solution $\omega_{o}$ are obtained. At iteration $j$, the design vector can be formulated as:

$$
\omega_{j+1}=\omega_{j}+h_{j}
$$

where $h_{j}$ is a function of the control and search direction parameters defined by BFGS technique as:

$$
\begin{aligned}
& h_{j}=\alpha_{j} y_{j} \\
& y_{j}=-\left[A_{j}\right]^{-1} \nabla f\left(\omega_{j}\right)
\end{aligned}
$$

where $y_{j}$ is the search direction component, $\left[A_{j}\right]$ is an approximation of the inverse Hessian matrix $[H]^{-1}$ and $\nabla f\left(\omega_{j}\right)$ is the gradient of the function.

The approximation to the Hessian matrix is further computed by [40]:

$$
\begin{aligned}
& {\left[A_{j+1}\right]=\left[A_{j}\right]+\left(1+\frac{v_{j}^{T}\left[A_{j}\right] v_{j}}{e_{j} v_{j}}\right) \frac{e_{j} e_{j}^{T}}{e_{j}^{T} v_{j}}-\frac{e_{j} v_{j}^{T}\left[A_{j}\right]}{e_{j}^{T} v_{j}}-} \\
& \frac{\left[A_{j}\right] v_{j} e_{j}^{T}}{e_{j}^{T} v_{j}}
\end{aligned}
$$

and

$v_{j}=\nabla f_{j+1}-\nabla f_{j}=\nabla f\left(\omega_{j+1}\right)-\nabla f\left(\omega_{j}\right)$

The proposed algorithm for throughput optimization employs the system of equations $f\left(\omega_{j}\right)$ derived from (16). The value of $f\left(\omega_{o}\right)$ is computed given the initial values of $\omega_{o}$ and $h_{o}$ in relation to (18) and (19) such that $\omega_{1}=\omega_{0}+h_{0}$ and the process is repeated till it converges to the optimal solution $\omega_{\text {opt }}$. The optimal value $\omega_{\text {opt }}$ converges at iteration $j$ when $\left|f\left(\omega_{j+1}\right)-f\left(\omega_{j}\right)\right|$ and $\left\|\nabla f\left(\omega_{j}\right)\right\|$ are less than the tolerance errors $\epsilon_{1}$ (tolerance of the function from previous iteration) and $\epsilon_{2}$ (tolerance on the gradient value) respectively. To prevent an infinite loop of iterations, the maximum allowable number of iteration is set to $j_{\max }$ such that the iteration stops if it does not converge to optimum value at $j=j_{\text {max }}$. 
Table 1. Proposed OFO-OFDM Algorithm for High Capacity Throughput Data Transmission

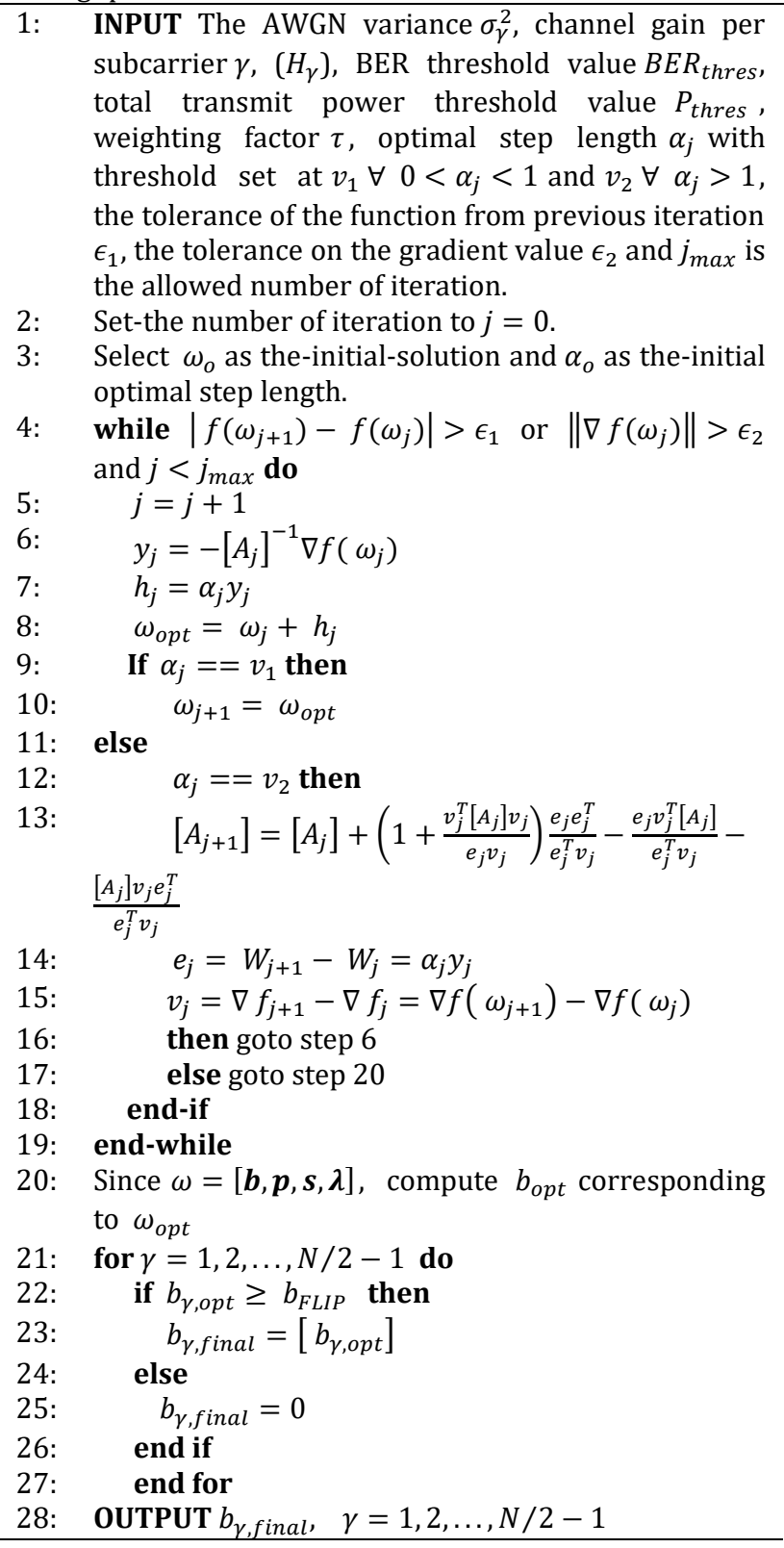

Hence, the process could be reinitiated and once the optimal value is attained at $\omega_{\text {opt }}$, the optimized throughput $b_{\gamma, o p t}$ is obtained. For the purpose of sign convention, the proposed scheme is referenced as Optimized Flipped Optical (OFO) OFDM scheme and the algorithm is presented in Table 1 . The multicarrier scheme considered is an optical-OFDM system with total number of $\mathrm{N}=1024$ subcarriers. The transmit signal $x$ is modelled as a Gaussian distribution with zero mean based on the central limit theorem as stipulated by [25], given that the transmitted time-domain samples will be approximately equivalent to a Gaussian distribution when the total number of subcarriers is sufficiently large, for $\mathrm{N} \geq 64$, and variance $\sigma^{2}$ with electrical and optical powers of $E\left(x^{2}\right)$ and $E(x)$ respectively. The channel impulse response and the power delay profile are derived from $[35,41]$ and the modulations (4QAM, 16QAM, 64QAM and 256QAM) supported by wideband standard [42] are adapted for the purpose of validation of the BER performance. The conventional multicarrier schemes as well as the proposed algorithm's simulation parameters are derived directly from the model's formulations. The threshold BER is set to $10^{-4}$ and threshold Power to $0.1 \mathrm{~mW}$ so as to constrain the performance of the proposed scheme relative to maximizing the throughput and to also align with standardization and best practices [43]. The BFGS algorithm parameters are defined as: $v_{1}=0.5, v_{2}=1, \epsilon_{1}=$ $\epsilon_{2}=10^{-6}$ and $j_{\max }=10^{2}$.

\section{Results}

The proposed high throughput signal conditioning scheme was simulated based on the mathematical and model-based outcome relative to the conventional signal conditioning schemes. The conventional optical schemes were first investigated to adapt the RF/wifi based OFDM for optical communication (VLC). These schemes took cognizance of the peculiarities of VLC applications since the signal had to be real valued and unipolar while maintaining intensity modulation/ direct detection [29]. In line with the related work, among the contending conventional schemes (DCO, ACO and FLIP), FLIP showed superiority in terms of energy efficiency while maintaining spectral efficiency for VLC application. While considering future projections for densification and data rate requirements, the throughput capacity of the schemes demands an upgrade which has led to this investigation. The FLIP scheme having less computational complexity and more power efficient among the contending conventional optical OFDM signal conditioning schemes [31] was optimized and simulated on MATLAB version 9.2 using the Lagrange multiplier to compute the stationary point for high throughput, while estimating the optimal throughput value using the Broyden-Fletcher-Goldfarb-Shanno algorithm. The proposed signal conditioning scheme performance was evaluated relative to the transmission parameters and signal-to-noise ratio. The power and spectral efficiencies of the conventional and proposed schemes are compared to ascertain the superiority of the proposed scheme.

In Figure 1, the DCO- time domain symbol is seen to be twice as large as those of the ACO, FLIP and the proposed (OFO) schemes since the dc-bias only shifts the entire signal amplitude of the electrical scheme to the positive amplitude scale without clipping one-half of its spectrum thereby making it spectrally efficient but power inefficient since a higher power value is required to initiate dc-bias. To improve on the power efficiency, one-half of the entire signal is clipped for the ACO- and flipped for the proposed (OFO), and FLIP- schemes without necessarily compromising 
data integrity since both halves contains the same information symbols with one-half mirroring the other half. This again is consistent with the validations in the literature.

Following directly from Figure 1 is the amplitude PDF and CDF shown in Figure 2 which further validates the fact that the optical OFDM schemes are real and unipolar covering the positive amplitude scale alone. The electrical signal covers both negative and positive amplitude scale since the processed signal is complex valued and bipolar.

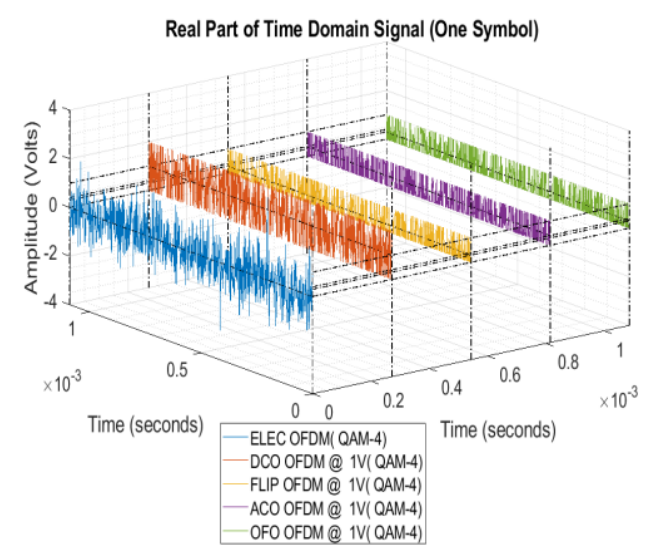

Figure 1. 3-D Plot of the Time Domain Signal of a Single Symbol of the Proposed (OFO), Electrical- and Conventional Optical- OFDM Schemes

Figure 2 depicts the Probability Density Function (PDF) and Cumulative Density Function (CDF) plot of the power and amplitude distribution of the proposed (OFO), electrical-, and the conventional optical schemes. For any given cumulative probability value, DCO, ACO, and the Electrical schemes require more power values relative to the proposed (OFO) and FLIP schemes. In evaluating the power cost of the schemes, for OFO and FLIP, there is $80 \%$ probability of being below 0.75 power values, whereas ACO-, Electrical and DCO are 80\% probability below 1, 1.25 and 3.25 respectively. The CDF and PDF of OFO- and FLIP overlap showing their superiority in terms of power and amplitude distribution and are more power efficient in comparison with their investigated counterpart. The Electrical, ACO and DCO schemes requires 1.7, 1.3 and 4.3 times more power respectively to drive the model in comparison with OFO and FLIP which poses a high cost on their implementation and design complexity. For the conventional schemes, FLIP is preferred due to its superior power efficiency and less complexity which has engendered its optimization in this work. The gradient of the CDF plot is the same as the PDF at those values.

Figure 3 shows the PDF and CDF of the proposed (OFO) algorithm using diverse signal amplitude voltage values and 4QAM modulation. We observed that the signal amplitude spans an equivalent range as the voltage value. All the modulation constellations are seen to have an equivalent pattern, according to the amplitude PDFs and CDFs shown. As the signal amplitude voltage increases, the scheme is seen to be more power efficient since there is higher percentage of the probability that the scheme will utilize less power.
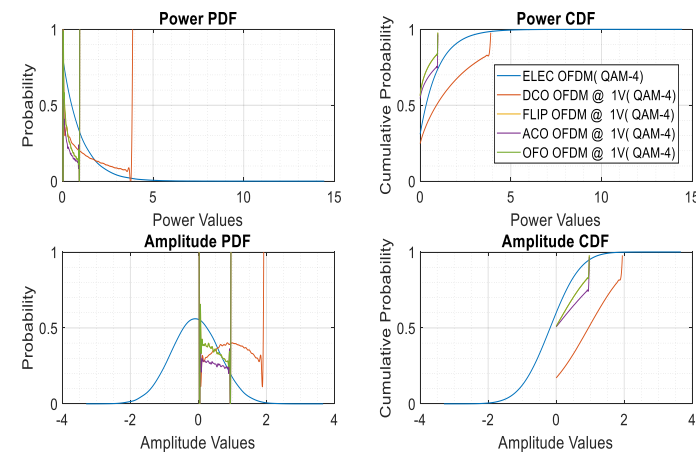

Figure 2. Probability Density Function (PDF) and Cumulative Density Function (CDF) of the Power and Amplitude of the Proposed (OFO), Electrical- and Conventional Optical- OFDM Schemes
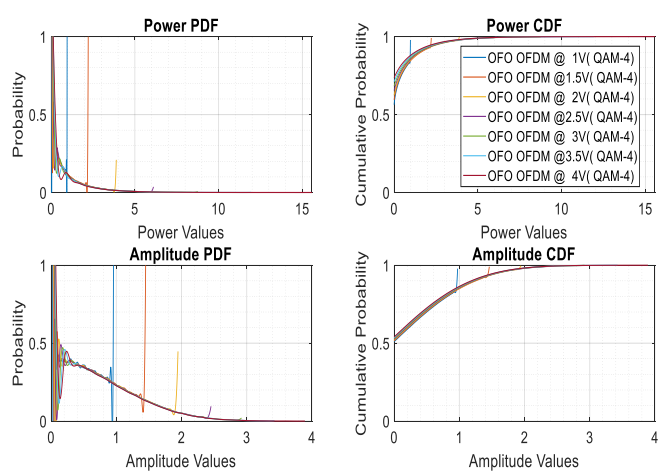

Figure 3. Comparison of Power and Amplitude Distribution of the Proposed (OFO) Signal Conditioning Scheme at Different Signal Amplitude Voltage

In relation to the power efficiency of the proposed scheme, to validate its spectral efficiency, the BER in relation to increasing SNR is presented. At increasing SNR values, for throughput maximization, higher order MQAM OFO could be utilized for symbol transmission despite the already validated $(4,16,64$ and 256) QAM. The constellation order of 512QAM and 1024QAM could achieve up to 9bits/symbol and $10 \mathrm{bits} /$ symbol respectively.

To justify the performance superiority of the proposed OFO scheme, the BER performance is validated by the four modulation constellations supported by wideband standard (4QAM, 16QAM, 64QAM and 256QAM) respectively [42]. The modulation constellations are required for bit mapping regardless of the nature of the signal (complex or real) being processed. Since the unipolarity and real valued signal has been maintained in VLC, its application follows directly 
from the fact that the signal of interest has been digitized making the wideband standard suitable for either RF/wifi or VLC. At higher SNR values, based on the findings in this research work, the performance of the proposed modulation constellations (512QAM and 1024QAM) at 3.5Volts are also presented. Figure 4 to Figure 9 compares the BER performances of the contending signal conditioning schemes based on various wideband modulations investigated in this study. Following the recommendation made for the selection of $1.5 \mathrm{~V} 4 \mathrm{QAM}$ subcarrier modulation at low SNR; OFO scheme is seen to outperform the conventional schemes in terms of lower BER as presented in Figure 4.

Figure 5 compares the BER performance of the contending schemes using 2V 16QAM subcarrier modulation. Their compared BER performances at $15 \mathrm{~dB}$ SNR showed that OFO, DCO, ACO and FLIP achieved $1.644 \times 10^{-3}, 1.833 \times 10^{-2}, 6.2 \times 10^{-2}$ and $3.179 \times 10^{-2}$ respectively. The proposed (OFO) algorithm is seen to achieve less BER at the specified SNR indicating its superiority over the conventional schemes.

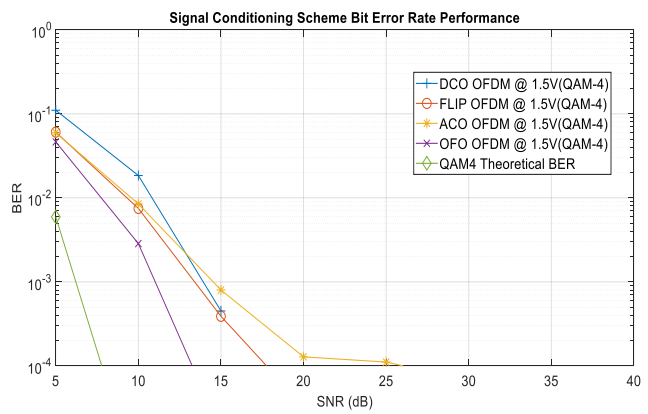

Figure 4. BER Performance of the 4QAM OFO and Conventional signal Conditioning Schemes for Wideband Application for 1.5Volts Signal Amplitude

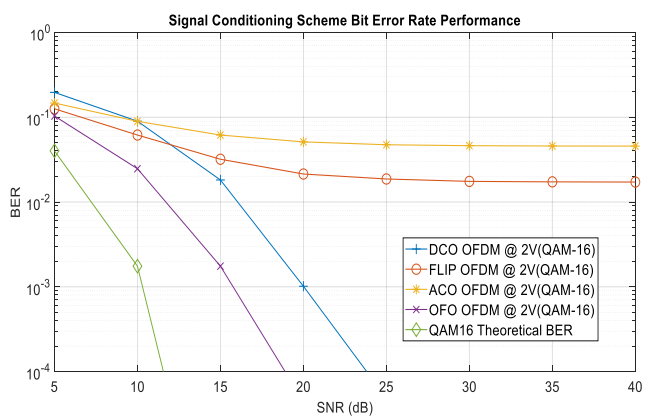

Figure 5. BER Performance of the 16QAM OFO and Conventional signal Conditioning Schemes for Wideband Application for 2Volts Signal Amplitude

Figure 6 compares the BER performances of the contending schemes using $2.5 \mathrm{~V}$ 64QAM subcarrier modulation. OFO, ACO, DCO and FLIP are seen to achieve BER of $1.439 \times 10^{-2}, 0.1825,8.237 \times 10^{-2}$ and $6.089 \times 10^{-2}$ at $15 \mathrm{~dB}$ respectively. The lower value of OFO BER implies performance superiority retative to the other schemes at comparable SNR.
The performcance of the recommended subcarrier modulation at $3 \mathrm{~V}$ being 256QAM is compared in relation to the contending signal conditioning schemes in Figure 7. At SNR of $20 \mathrm{~dB}$, the BER performance of OFO, FLIP, DCO and ACO are $3.722 \times 10^{-4}, 4.691 \times 10^{-2}, 2.228 \times 10^{-2}$ and 0.2751 respectively. OFO is see to surpass the conventional techniques in terms of BER performance.

Comparing the evaluated performances with the BER-SNR plot in Figure 7, FLIP outperforms DCO because of its lower BER performance at lower SNRs between 5 to $21.5 \mathrm{~dB}$.

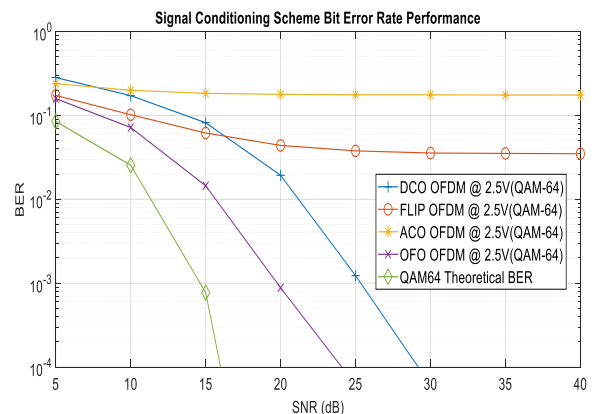

Figure 6. BER Performance of the 64QAM OFO and Conventional signal Conditioning Schemes for Wideband Application for 2.5Volts Signal Amplitude System

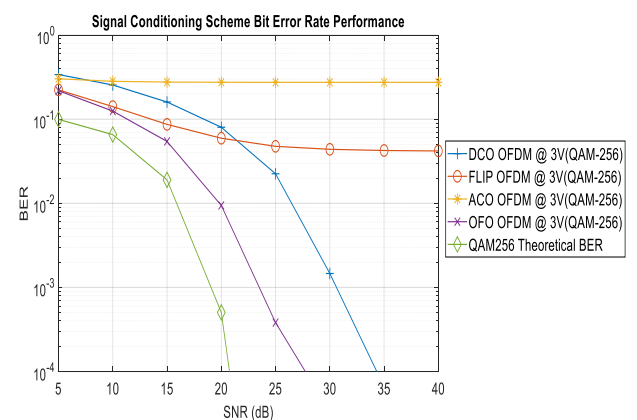

Figure 7. BER Performance of the 256QAM OFO and Conventional signal Conditioning Schemes for Wideband Application for 3Volts Signal Amplitude System

In Figure 8, OFO outperforms the conventional schemes with lower BER over comparable values of SNR at 3.5V signal amplitude for 512QAM subcarrier modulation. At 25dB, OFO, FLIP, DCO and ACO achieved BER of $9.926 \times 10^{-4}, 2.63 \times 10^{-2}, 5.612 \times 10^{-2}$ and 0.2962 respectively. OFO shows an appreciable improvement in BER performance relative to the conventional schemes.

Figure 9 depicts the BER performance measure of the contending signal conditioning schemes. The BER performance of OFO, FLIP, DCO and ACO at 30dB SNR for $3.5 \mathrm{~V}$ 1024QAM subcarrier modulation are $3.156 \times 10^{-4}, 4.197 \times 10^{-2}, 2.765 \times 10^{-2}$ and 0.3218 respectively. The BER response of the OFO shows superiority over the conventional techniques at comparable SNR. FLIP gives lower errors in comparison with DCO because of its lower BER at SNR between $5 \mathrm{~dB}$ and $27.5 \mathrm{~dB}$. 


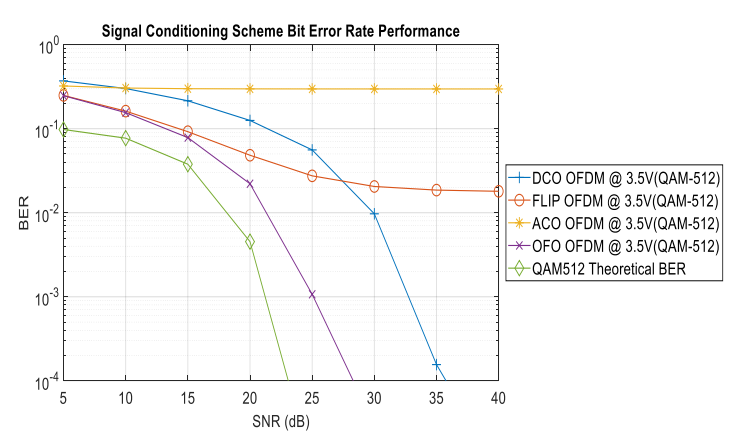

Figure 8. BER Performance of the 512QAM OFO and Conventional signal Conditioning Schemes for Wideband Application for 3.5Volts Signal Amplitude System

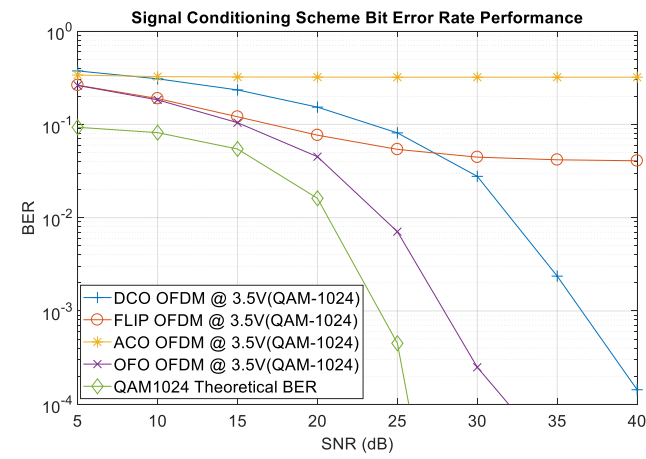

Figure 9. BER Performance of the 1024QAM OFO and Conventional Signal Conditioning Schemes for Wideband Application for 3.5Volts Signal Amplitude System

Figure 10 shows the plot of the average throughput and average SNR with and without power constraint at $\tau=0.5$. The average throughput is seen to increase as the average SNR increases. Based on the deductions made in Figure 4 to Figure 9, at SNR beyond $25 \mathrm{~dB}$, the selection of higher modulation constellations such as 512QAM and 1024QAM will result in higher throughput since more subcarriers are used at higher SNR values. At SNR greater than $25 \mathrm{~dB}$, the OFO algorithm will keep the average transmit power constant as a measure for ensuring power efficiency while maximizing the average throughput since all the subcarriers are practically in use. When power constraint is imposed, a slight reduction in the average throughput is seen at higher SNR and with comparable performance at lower SNR. The reason for this is that at low SNR, less power is required such that the total transmit power is below the power constraint so that the average throughput with and without power constraint are comparable. As SNR increases, the subcarrier power allocation also increases which results in a slight reduction in the average throughput as the total subcarrier transmit power exceed the imposed constraint power which justifies the claim that OFO satisfies the power constraint in maximizing the throughput. Based on the mathematical formulation in (15) and (16), increasing the value of the weighting factor $\tau$ reduces the average throughput since higher values of $\tau$ provides less weight for throughput maximization without power constraint, thereby ultimately reducing the average throughput as depicted in
Figure 11. With power constraint, the average throughput is seen to saturate when the imposed power constraint is exceeded and gradually reduces with increasing $\tau$. Hence, $\tau$ is necessary for channel adaptation in terms of throughput requirement for a given wireless connection.

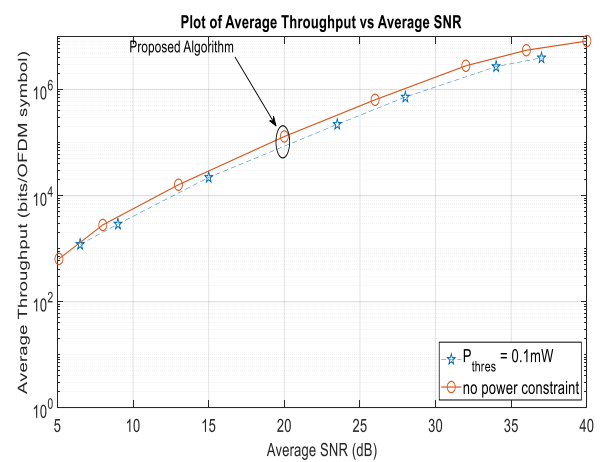

Figure 10. Average Throughput Performance of the Proposed Algorithm (OFO) at $\tau=0.5$ in relation to Average SNR with and without Power Constraint

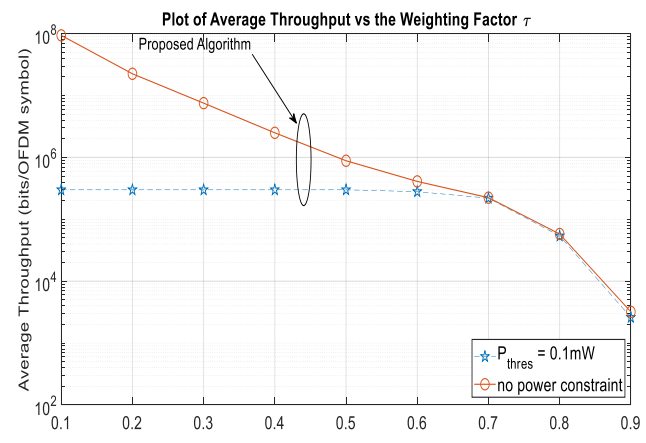

Figure 11. Average Throughput Performance of the Proposed Algorithm (OFO) in relation to the Algorithmic Weighting Factor $\tau$ at $\sigma_{\gamma}^{2}=10^{-3} \mu \mathrm{W}$ with and without Power Constraint

Figure 12 depicts the plot of average throughput and the threshold power characterized by an appreciable increase in average throughput as the imposed constraint power increases largely because the total transmit power is usually normalized to the threshold power such that increasing the threshold power will literally result in an increase in the average throughput. At higher values of the threshold power, the total subcarrier transmit power is constrained by the threshold power to the extent that transmission is done at near constant power which results in a corresponding near constant average throughput at higher threshold power.

Figure 13 compares the average throughput performance of the proposed algorithm and the existing conventional algorithms. The proposed algorithm (OFO) is seen to outperform the conventional optical techniques relative to increasing SNR. Among the conventional techniques, DCO is seen to be more spectrally efficient in comparison with ACO and FLIP largely because more power is required to drive the scheme as well as the utilization 
of the entire subcarriers. Although DCO is spectrally efficient, it has been shown in Figure 3 to be power inefficient and as such the complexity in its implementation is much higher. Among the contending algorithms, ACO and FLIP show comparable average throughput performance with respect to increasing SNR. At low SNR, FLIP technique is seen to outperform ACO up to $15 \mathrm{~dB}$ whereas ACO achieves a higher average throughput at higher SNR since the percentage probability that ACO will require more power increases slightly in comparison with FLIP as shown in Figure 3. This further informs the choice of FLIP over ACO and DCO among the contending conventional techniques based on its tradeoff between power and spectral efficiency. Since less power is required in FLIP to achieve comparable average throughput in relation to ACO, its design complexity is less. Although OFO and FLIP shows similarity in power handling, the adaptive capability of OFO guarantees enormous improvement over the conventional techniques in achieving a much higher average throughput.

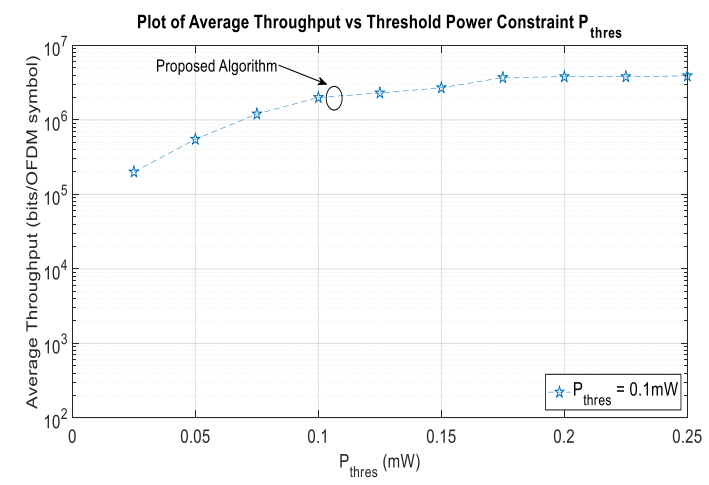

Figure 12. Average Throughput Performance of the Proposed Algorithm (OFO) in relation to the Power Constraint $P_{\text {thres }}$ at $\sigma_{\gamma}^{2}=10^{-3} \mu \mathrm{W}$ and $\tau=0.5$

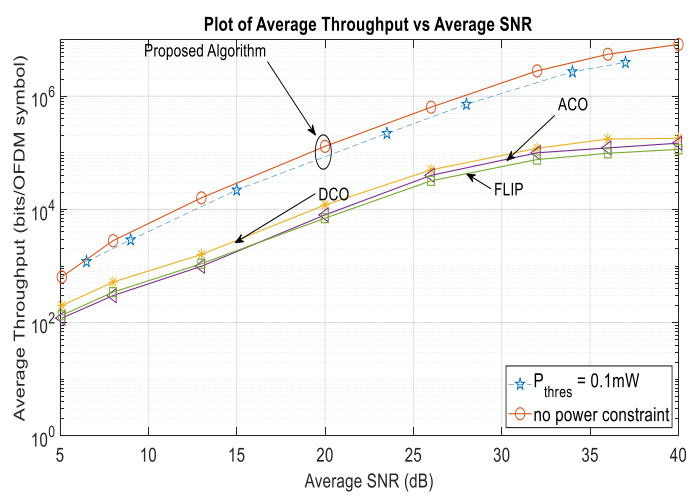

Figure 13. Comparison of the Average Throughput Performance of the Proposed Algorithm (OFO) and the Conventional Optical Signal Conditioning Schemes

The proposed OFO algorithm is further validated by comparing the average throughput performance relative to the average throughput of its adaptive radio frequency counterparts in [35, 42, 44] under comparable operating conditions. To model similar operating condition, the transmit power used in [35, $42,44]$ was estimated by equitable distribution of the allocated average transmit power in the proposed OFO algorithm to the total number of subcarriers. Result in Figure 14 and Table 2 showed that the proposed algorithm outperformed the existing algorithms with an appreciable improvement in the average throughput.

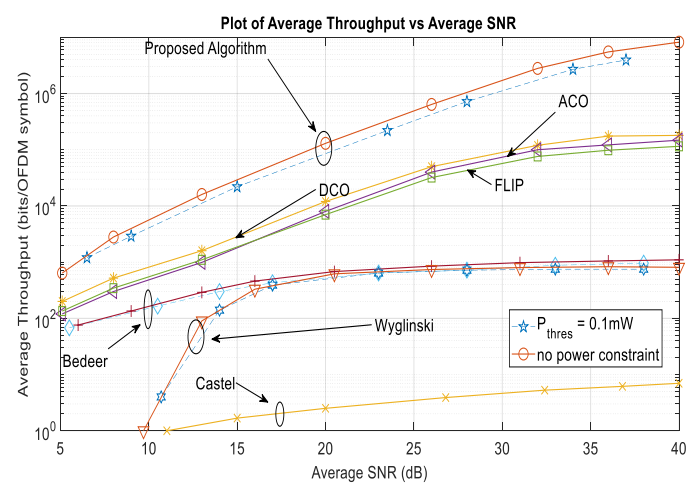

Figure 14. Validation of the Average Throughput Performance of the Proposed Algorithm (OFO) as a function of the Average SNR

Table 2. Performance Comparison of the Algorithms for Validation

\begin{tabular}{ccccccc}
\hline $\begin{array}{c}\text { Castel } \\
\text { (dB) }\end{array}$ & $\begin{array}{c}\text { Wyglinski } \\
\text { (dB) }\end{array}$ & $\begin{array}{c}\text { Bedeer } \\
\text { (dB) }\end{array}$ & $\begin{array}{c}\text { FLIP } \\
\text { (dB) }\end{array}$ & $\begin{array}{c}\text { ACO } \\
\text { (dB) }\end{array}$ & $\begin{array}{c}\text { DCO } \\
\text { (dB) }\end{array}$ & $\begin{array}{l}\text { OFO } \\
\text { (dB) }\end{array}$ \\
1.89 & 2.86 & 2.99 & 3.45 & 3.45 & 3.46 & 3.59 \\
\hline
\end{tabular}

\section{Discussion and Conclusion}

A novel signal conditioning scheme for high throughput application in visible light frequency spectrum has been presented in this article. The approach to throughput enhancement was based on the dynamic adaptation of the transmission parameters to the channel condition in relation to the bit error rate (BER) performance using Lagrange Multiplier and Broyden Fletcher Goldfarb Shanno algorithm. The study showed that 512QAM and 1024QAM with 512 and 1024 possible signal combination and effective symbol throughput of 9bits/symbol and 10bits/symbol respectively could be included for wideband application in visible light communication at SNR beyond $25 \mathrm{~dB}$. It was also observed that the conventional ACO and FLIP schemes cannot be employed for communication systems in most use cases due to the abrupt deterioration of the BER response which can be traceable to the impact of the channel noise on the schemes. The proposed OFO scheme which adaptively manages the channel effect was seen to be more robust relative to the BER response with an overall enhanced throughput being its observed merit. Table 2 follows directly from Figure 14 and depicts an appreciable performance increase of $1.89 \mathrm{~dB}, 2.86 \mathrm{~dB}$ and $2.99 \mathrm{~dB}$ for Castel, Wyglinski and Bedeer respectively while FLIP, ACO, DCO and OFO showed an improvement of $3.45 \mathrm{~dB}, 3.45 \mathrm{~dB}, 3.46 \mathrm{~dB}$ and $3.59 \mathrm{~dB}$ respectively. From the foregoing, the proposed OFO algorithm is seen to outperform the 
spectrally efficient DCO scheme by about $3 \%$ increment in throughput performance while outperforming the energy efficient FLIP and ACO schemes by $4 \%$ respectively. The proposed OFO scheme is also seen to outperform the RF-based adaptive schemes with an appreciable margin. OFO outperformed the Castel, Wyglinski and Bedeer algorithms by a percentage increase of $63 \%, 24 \%$ and $19 \%$ respectively. The proposed algorithm which maximizes the throughput with constraint on the total transmit power and average bit error rate (BER) showed performance superiority at increasing average signal-to-noise ratio (SNR) when compared with Castel, Wyglinski and Bedeer algorithms. The algorithm also outperformed the existing conventional signal conditioning schemes (DCO, ACO and FLIP) with a low bit error rate (BER) threshold value. This study will be relevant to the communication sector in proposing a high performance signal conditioning scheme and standardization for high throughput deployment in visible light communication.

\section{Acknowledgement}

The authors gratefully acknowledge the sponsorship of this project by the Petroleum Technology Development Fund (PTDF) under the grant award number P4567720076521527.

\section{Declaration of Ethical Code}

In this study, we undertake that all the rules required to be followed within the scope of the "Higher Education Institutions Scientific Research and Publication Ethics Directive" are complied with, and that none of the actions stated under the heading "Actions Against Scientific Research and Publication Ethics" are not carried out.

\section{References}

[1] Bernard J T Mallinder. 1988. Specification Methodology Applied to the GSM System. IEEE 8th European Conference on Electrotechnics, Conference Proceedings on Area Communication, Stockholm, Sweden, 458 - 461.

[2] Chen Shanzhi, Sun Shaohui, Wang Yinmin, Xiao Guojun, and Rakesh Tamrakar. 2015. A Comprehensive Survey of TDD-Based Mobile Communication Systems from TD-SCDMA 3G to TDLTE( A) 4G and 5G directions. IEEE China Communications, 12(2), 40 - 60.

[3] Shanzhi Chen and Jian Zhao. 2014. The requirements, challenges, and technologies for $5 \mathrm{G}$ of terrestrial mobile telecommunication. IEEE Communications Magazine, 52(5), 36 - 43.

[4] Augustus E Ibhaze, Patience E Orukpe, and Frederick O Edeko. 2020. Li-Fi Prospect in Internet of Things Network. FICC2020, Advances in Intelligent Systems and Computing. Cham, Switzerland: Springer Nature Switzerland AG, 1129, 272-280.

[5] Suneel Kumar, Prabha Tomar, and Aasheesh Shukla. 2015. Effectiveness of OFDM with Antenna Diversity. IEEE International Conference on Communication, Control and Intelligent Systems, Mathura, India, 172 - 175.

[6] Hai Thanh Vo, Shinya Kumagai, Tatsunori Obara, and Fumiyuki Adachi. 2013. Analog SingleCarrier Transmission with frequency-domain Equalization. IEEE 9th Asian-Pacific Conference on Communication, Denpasar, Indonesia, 698 702.

[7] Sudershan Mukherjee and Saif Khan Mohammed. 2016. Impact of Frequency Selectivity on the Information Rate Performance of CFO Impaired Single-Carrier Massive MUMIMO uplink. IEEE Wireless Communication Letters, 5(6), 1-4.

[8] H Sari, G Karam, and I Jeanclaud. 1994. Frequency-domain equalization of mobile radio and terrestial broadcast channels. IEEE GLOBECOM, San Francisco, CA, USA, 1-5.

[9] Dirk Slock. 2008. Diversity-Multiplexing Tradeoff simplified Receivers for frequencyselective MIMO Channels. IEEE 16th European Signal Processing Conference, Lausanne, Switzerland, 1-5.

[10] A. E Ibhaze, P. E Orukpe and F. O Edeko. 2020. Visible Light Channel Modeling for High-data Transmission in the Oil and Gas Industry. Journal of Science and Technology, 12(2), 46-54.

[11] David Falconer, Sirikiat Lek Ariyavisitakul, Anader Benyamin-Seeyar, and Brian Eidson. 2002. Frequency domain equalization for singlecarrier broadband wireless systems. IEEE Communications Magazine, 40(4), 58 - 66.

[12] Hikmet Sari, Georges Karam, and Isabelle Jeanclaude. 1995. Transmission techniques for digital terrestrial TV broadcasting. IEEE Communications Magazine, 33(2), 100 - 109.

[13] S H Han and J H Lee. 2005. An overview of peakto-average power ratio reduction technique for multicarrier transmission. IEEE Wireless Communication, 12(2), 56-65.

[14] Charles U Ndujiuba, Oluyinka Oni, and Augustus E Ibhaze. 2015. Comparative Analysis of Digital Modulation Techniques in LTE 4G Systems. Journal of Wireless Networking and Communications, 5(2), 60-66.

[15] J. A. C. Bingham. 1990. Multicarrier modulation for data transmission: an idea whose time has come. IEEE Communications Magazine, 28(5), 5 $-14$. 
[16] Harald Witschnig, Alois Koppler, Andreas Springer, Robert Weigel, and Mario Huemer. 2002. A Comparison of an OFDM System and a Single Carrier System Using Frequency Domain Equalization. European Transactions on Telecommunications banner, 13(5), 519-530.

[17] Zhaocheng Wang, Qi Wang, Wei Huang, and Zhengyuan Xu. 2017. Visible Light Communications: Modulation and Signal Processing. New Jersey: John Wiley \& Sons, Inc.

[18] Oboyerulu E Agboje, Olabode B Idowu-Bismark, and Augustus E Ibhaze. 2017. Comparative Analysis of Fast Fourier Transform and Discrete Wavelet Transform Based MIMO-OFDM. International Journal on Communications Antenna and Propagation (I.Re.C.A.P.), 7(2), 168 - 175.

[19] Charles U Ndujiuba and Augustus E Ibhaze. 2016. Dynamic Differential Modulation of Subcarriers in OFDM. Journal of Wireless Networking and Communications, 6(1), 21-28.

[20] A Czylwik. 1997. Comparison between adaptive OFDM and single carrier modulation with frequency domain equalization. IEEE 47th Vehicular Technology Conference. Technology in Motion, Phoenix, AZ, USA, 2, 865 - 869.

[21] A Czylwik. 1998. Synchronization for single carrier modulation with frequency domain equalization. VTC '98. 48th IEEE Vehicular Technology Conference. Pathway to Global Wireless Revolution (Cat. No.98CH36151), Ottawa, Ont., Canada , 3, 2277 - 2281.

[22] P. F. M. Smulders and H. T. Muskens. 1993. Performance of decision feedback equalization in MM-wave indoor radio systems. Proceedings of 2nd IEEE International Conference on Universal Personal Communications, Ottawa, Ontario, Canada, 890 - 893.

[23] N Benvenuto and S Tomasin. 2002. On the comparison between OFDM and single carrier modulation with a DFE using a frequencydomain feedforward filter. IEEE Transactions on Communications, 50(6), 947 - 955.

[24] Zhaocheng Wang, Tianqi Mao, and Qi Wang. 2017. Optical OFDM for visible light communications. IEEE 13th International Wireless Communications and Mobile Computing Conference (IWCMC), Valencia, Spain, 1190 - 1194.

[25] J Armstrong. 2009. OFDM for optical communications. IEEE Journal of Light Wave Technology, 27(3), 189 - 204.

[26] J Armstrong and A J Lowery. 2006. Power Efficient Optical OFDM. IEEE Electronics Letters, 42(6), 370 - 372.
[27] Dobroslav Tsonev, Sinan Sinanovic, and Harald Haas. 2012. Novel Unipolar Orthogonal Frequency Division Multiplexing (U-OFDM) for Optical Wireless. IEEE 75th Vehicular Technology Conference (VTC Spring), Yokohama, Japan, 1 - 5.

[28] A. E Ibhaze, P. E Orukpe and F. O Edeko. 2020. High Capacity Data Rate System: Review of Visible Light Communications Technology. Journal of Electronic Science and Technology.

[29] A. E Ibhaze, F. O Edeko and P. E Orukpe. 2021. Comparative Analysis of Optical Multicarrier Modulations: An Insight into Machine Learningbased Multicarrier Modulation. Gazi University Journal of Science.

[30] Seong Taek Chung and Andrea J. Goldsmith. 2001. Degrees of Freedom in Adaptive Modulation: A Unified View. IEEE Transactions on Communications, 49(9), 1561 - 1571.

[31] Liang Wu, Zaichen Zhang, Jian Dang, Jiangzhou Wang, and Huaping Liu. 2016. Polarity Information Coded Flip-OFDM for Intensity Modulation Systems. IEEE Communications Letters, 20(8), 1089 - 7798.

[32] Jie Lian, Yan Gao, and Dianbin Lian. 2019. Variable Pulse Width Unipolar Orthogonal Frequency Division Multiplexing for Visible Light Communication Systems. IEEE Access, 7, 31022 - 31030 .

[33] Simeng Feng, Rong Zhang, Wei Xu, and Lajos Hanzo. 2019. Multiple Access Design for UltraDense VLC Networks: Orthogonal vs NonOrthogonal. IEEE Transactions on Communications, 67(3), 2218 - 2231.

[34] Kaiming Liu, Bihua Tang, and Yuan'an Liu. 2009. Adaptive Power Loading Based on Unequal-BER Strategy for OFDM Systems. IEEE Communications Letters, 13(7), 474 - 476.

[35] Ebrahim Bedeer, Octavia A. Dobre, Mohamed H. Ahmed, and Kareem E. Baddour. 2012. Optimal bit and power loading for OFDM systems with average BER and total power constraints. IEEE Global Communications Conference (GLOBECOM), Anaheim, CA, USA, 3685 - 3689.

[36] Igor Griva, Stephen G. Nash, and Ariela Sofer. 2009. Linear and Nonlinear Optimization. Philadelphia: Society for Industrial and Applied Mathematics.

[37] Edwin K. P. Chong and Stanislaw H. Zak. 2013. An Introduction to Optimization. New Jersey: John Wiley \& Sons, Inc.

[38] Rajesh Kumar Arora. 2015. OPTIMIZATION Algorithms and Applications. London: Taylor \& Francis Group, LLC. 
[39] Randy L. Haupt and Douglas H. Werner. 2007. Genetic Algorithms in Electromagnetics. Canada: IEEE Press (A John Wiley \& Sons, Inc.).

[40] Singiresu S. Rao. 2009. Engineering Optimization Theory and Practice. Canada: John Wiley \& Sons, Inc.

[41] Hai Lin, Xianbin Wang, and Katsumi Yamashita. 2008. A Low-Complexity Carrier Frequency Offset Estimator Independent of DC Offset. IEEE Communication Letters, 12(7), 520 - 522.

[42] Thijs Castel et al. 2016. Adaptive subcarrier modulation for indoor public safety body-tobody networks. IEEE 10th European Conference on Antennas and Propagation (EuCAP), Davos, Switzerland, 1-5.
[43] Xuan Huang, Fang Yang, Hailong Zhang, Jun Ye, and Jian Song. 2019. Subcarrier and Power Allocations for Dimmable Ehanced ADO-OFDM with Iterative Interference Cancellation. IEEE Access, 7, 28422 - 28435.

[44] Alexander M. Wyglinski, Fabrice Labeau, and Peter Kabal. 2005. Bit Loading With BERConstraint for Multicarrier Systems. IEEE Transactions on Wireless Communications, 4(4), 1383 - 1387. 